\title{
On the Hegemony of Naturalized Violence: An Apology
}

\author{
Angela Willey $^{1} \cdot$ Banu Subramaniam $^{1}$
}

Received: 11 June 2017 / Accepted: 13 June 2017 / Published online: 7 July 2017

(C) Springer Science+Business Media, LLC 2017

We wrote a Commentary on van Anders (2015) "Beyond Sexual Orientation," to which we added the title "Fighting the Derpy Science of Sexuality" (2016); regrettably, we did so without adequate research on the term "derpy." In their response to our Commentary, van Anders and Schudson (2017) call us out for the use of this "ablist slur" in our title.

The word "derpy" was introduced to us as a pop-cultural term that meant believing in something despite the fact that it has been disproven. A provocative notion, given that our aim was to suggest that while queer theory has effectively undone "sexuality" as a concept, empirical research (even thoughtful, feminist sexual science) persists as if the complexity of sexuality is ultimately map-able and as if the right set of variables will resolve the epistemological differences of critical theory and neuroscience once and for all. This is not an individual or even disciplinary failing, but rather an effect of a distribution of epistemic authority that privileges certain frames and ways of knowing. We-as a culture and the science that that culture produces-seem to believe, despite good evidence of historical, cultural, and in-group variation as well as trenchant analyses of the political work of sexuality as a concept, that sexual science can reveal what Foucault calls "the truth of the self." Our aim was to ask what questions we might ask if we begin from the insights of queer studies about sexuality. In other words, what rich capacities does "sexuality" serve as proxy for? What are we measuring? What might we hope to know about bodies and pleasures if we eschew sexuality as such?

Angela Willey

awilley@umass.edu

1 Department of Women, Gender, Sexuality Studies, University of Massachusetts Amherst, W401 South College, 150 Hicks Way, Amherst, MA 01003-9274, USA
As it turns out, the term "derpy" has also been appropriated as an ablist slur. We regret our negligence in not figuring that out before the Commentary was published. That in our reach to find pithy and accessible language that destabilizes automatic epistemic authority we grasped a term whose usage is deeply implicated in ablist white supremacist legacies of the science of intelligence oddly mirrors one point of our Commentary. The pervasiveness of naturalized assumptions about difference permeates our knowledge projects, despite our best hopes and intentions. "Sexuality," like "intelligence," is, in part, an artifact of racial science and the eugenic control of populations. Knowing that, how do we broach its materiality? We would like to see the concepts themselves utterly problematized and the phenomena they measure more carefully empirically examined and described. "A comprehensive theory of sexuality" has its strategic perks, but necessarily falls short of that queer ambition. In this spirit of undoing the categories we have inherited, please accept our apologies for the title in question. ${ }^{1}$

\section{References}

Subramaniam, B., \& Willey, A. (2016). Fighting the derpy science of sexuality [Commentary]. Archives of Sexual Behavior, 45, 513-515. van Anders, S. M. (2015). Beyond sexual orientation: Integrating gender/sex and diverse sexualities via sexual configurations theory. Archives of Sexual Behavior, 44, 1177-1213.

van Anders, S. M., \& Schudson, Z. C. (2017). A response to commentaries on "Beyond Sexual Orientation: Integrating Gender/Sex and Diverse Partnered Sexualities via Sexual Configurations Theory" (van Anders, 2015). Archives of Sexual Behavior. doi:10.1007/s 10508-017-1004-7.

\footnotetext{
${ }^{1}$ Editor's note: For the interested reader, I suggest that you GOOGLE "Is the word derpy offensive?" and draw your own conclusions.
} 